\title{
Boundary Elements from 1960 to the Present Day
}

\author{
J O Watson \\ School of Mining Engineering, \\ University of New South Wales, Australia
}

\begin{abstract}
The development of boundary element methods as seen by one researcher in the field is described, from publication of the first results in 1963 to the present day. Details are given of the circumstances in which research was carried out. The objectives of the research are outlined, and the strategy according to which progress was made towards those objectives is explained. An assessment is made of what has actually been achieved, and topics for future research are proposed.
\end{abstract}

\section{Introduction}

The approximate numerical solution of boundary value problems for partial differential equations became a practical proposition towards 1960, as advances in solid state circuitry made it possible to construct reasonably reliable computers with enough memory to store thousands of instructions and items of data, and the first high-level programming languages were developed. It was natural to replicate on the computer the existing procedures of hand computation, and among the first to consider the use of computers were structural engineers who were familiar with matrix and iterative methods of analysis of frameworks. The idea of a one dimensional structural member was generalised to an element of a multidimensional continuum, and so it was that finite elements for solid mechanics came into being.

The first researchers to propose the solution of a boundary integral equation were mathematicians. By the time their work was published, the development of the finite element method was well under way. Furthermore, finite elements are relatively easy to develop, and before long some quite general software packages were available. However, some determined individuals struck out in a different direction, and attempted to turn the boundary integral equation method into a practical means of engineering analysis. This paper is a brief account of the efforts of a few of those researchers during the past forty years. 


\section{Origins}

For practical purposes, the development of boundary element methods began with the work of Jaswon, Ponter and Symm [1, 2, 3] of the Department of Mathematics at Imperial College, London. In their solutions of boundary value problems for Laplace's equation in two dimensions, these authors adopted what has come to be known as the direct formulation, in which the function to be computed and the fundamental solution (Green's function for an infinite domain) are substituted into Green's symmetric identity to yield a boundary integral equation. The numerical implementation was very simple, with the potential and its normal derivative taken to be uniform over each boundary segment, but nevertheless the method was shown to be a promising alternative to finite elements and finite differences. By 1963 when this work was published, though, attention was already focused on finite elements and in any case few engineers understood anything about integral equations. Despite the clarity with which Jaswon et al presented their ideas, this early work was largely ignored.

\section{Research at Southampton}

I was introduced to boundary integral equations in 1966, as a research student at the University of Southampton under Hugh Tottenham of the Department of Civil Engineering. Of the research topics on offer, I chose the analysis of stress in prestressed concrete pressure vessels for gas cooled nuclear reactors. Tottenham possessed a remarkable library of Soviet books, and encouraged research students to explore possible applications of the work of Muskhelishvili [4], Kupradze [5] and others. I laboured through Muskhelishvili's complex variable theory, and struggled with Kupradze's tortuous mathematical notation without the benefit of being able to read the text. I eventually understood that the desired three dimensional elastic analysis could be reduced to the solution of a singular boundary integral equation for a fictitious force density function, followed by the computation of physically meaningful results from the proposed integral representation of displacement. This was the indirect method: I had read the papers by Jaswon et al, but failed to recognise that their approach was the more practical.

At that stage I became fascinated and somewhat preoccupied by the mathematical detail. This was not altogether unproductive, as I pondered among other things the problems posed by edges and corners and came to understand that the fictitious force densities would probably tend to infinity near such features. My attempts to determine the nature of the supposed singularities, however, were unsuccessful. Rather late in the day, I turned to the question of numerical implementation. The university had an excellent computer, an ICL1905E, capable of somewhere between 200000 and 400000 floating point operations per second and very reliable too. Departmental policy was that software be written in Algol. By my association with students researching into finite elements I knew of interpolation using shape functions, and Tottenham had suggested that Gaussian quadrature formulae [6] could be of interest, but I failed to see the relevance of these techniques at the time. The guiding principle was to be that, since the dimension of the numerical problem was reduced from three to two, the 
boundary integral method would outperform finite elements without recourse to such sophistication.

Contemporaries at Southampton included Banerjee, who applied a specialised version of the indirect method to the analysis of pile groups, and Brebbia who worked on finite elements for shells but later became a proponent of boundary element methods: indeed, it has been said that Brebbia was the inventor of the term 'boundary element'.

\section{Early outcomes}

The method as implemented at Southampton did not live up to expectations. My research [7] was completed during spare time whilst I worked in London as an engineering applications programmer (in FORTRAN). At least the lesson had been learned that the much-vaunted reduction of the dimension of the numerical problem would not by itself assure the success of boundary element methods.

The work of Jaswon et al had been ignored in UK, but in USA it was taken up by Rizzo [8] for plane strain of an elastic material and then by Cruse [9] for the general three dimensional case. The starting points for Rizzo's work are Betti's theorem which is analogous to Green's symmetric identity, and the fundamental solution for plane strain. Rizzo presents a useful discussion of such theoretical aspects as conditions for existence of a solution, and notes the need to evaluate a Cauchy principal value in the boundary integral equation of elastostatics. He points out that his cautious approach to numerical implementation is intended only as a demonstration of feasibility, and that computational efficiency could probably be improved by mesh gradation. Concern is expressed about the possibility of ill-conditioning of simultaneous equations.

In three dimensional analysis undoubtedly inspired by the work of Rizzo, Cruse approximates the surface by plane triangular elements, over each of which displacement and traction are taken to be uniform. In the system of simultaneous equations for displacement and traction, coefficients of traction are scaled so that all equation coefficients are in the same order of magnitude, to avoid ill-conditioning. Whereas Rizzo generally computes equation coefficients by Simpson's rule, Cruse evaluates them analytically. Displacement and stress at interior points, though, are still computed by numerical integration.

By 1970, there was interest not just in proving that boundary element methods work, but in making them work better. A first step in that direction was taken by Riccardella [10], who developed a program for the analysis of plane strain in which displacement and traction were taken to vary linearly over each element. The price to be paid for improved accuracy and computational efficiency was more complicated integration, and new logic to accommodate the possibility that nodes at which the integral equation is written were located at corners of the boundary. That possibility now arose because nodes were at the ends of elements, instead of at the midpoints. The elements were still straight, so relatively large numbers of them were required to model curved boundaries. Riccardella's program with the addition of joint elements lives on today in 
Australia, as BITEMJ. Attempts were also made to broaden the scope of the method. Given the difficulties of application of the finite element method to problems of fracture mechanics, Cruse and others began to consider how boundary elements might be used to analyse cracks, for which boundary element methods in their basic form yield more unknowns than equations.

\section{Centre Technique des Industries Mécaniques}

Towards the end of 1971 I was contacted by Brebbia, who asked that I pay him an overnight visit to discuss the implementation of boundary element methods with his external Ph.D student, Jean-Claude Lachat. Grudgingly, I obliged. Lachat discussed with me through Brebbia as interpreter some aspects of boundary elements, and then produced a job application form, requesting that I reply to his offer within four weeks. The prospect of life in France did not greatly appeal, but given the salary offered and the shaky financial state of the company for which I was working in London I decided to accept.

Lachat, a mathematician and keen sportsman, was head of the Département Théorique et Engrenages, a group of about ten engineers who developed software and acted as consultants to the mechanical engineering industry. Funds had been allocated for the development of finite element and boundary element programs for stress analysis in three dimensions. During my first year with CETIM, I wrote a program for three dimensional elastic finite element analysis. My experience as a programmer in London served me well. There were awkward times at first, as Lachat did not take kindly to my strategy of designing and writing a program in its entirety before testing any of it, and did not understand why I spent so much time incorporating checks on the validity of input data, and automatic data generation facilities. The language barrier, or the pretence of a language barrier, was useful in the early days.

At the beginning of 1973 I started work on boundary elements. I was to develop firstly a program for plane strain, then one for three dimensional analysis. Lachat knew of the work of Rizzo and Cruse, and proposed that the direct formulation be used. I readily agreed, having met Cruse in 1972 and discussed with him the direct and indirect approaches. The finite element programming had been most instructive in respect of shape functions, Gaussian quadrature and out-of-core simultaneous equation solution techniques. It seemed clear that the boundary elements should be isoparametric, with at least quadratic variation so that curved surfaces could be modelled accurately. Analytical integration was then out of the question, and Gaussian quadrature was far superior to Simpson's rule. Adaptations of Gaussian quadrature could integrate weakly singular functions, but Cauchy principal values could not be computed directly by quadrature. If problems of practical size were to be analysed, the simultaneous equation coefficients would have to be held on disc.

Lachat did not agree with this view, or perhaps he considered the proposed strategy too adventurous. I designed the program so that once it had been shown to work with constant elements it could easily be converted to my proposed specification. All went 
according to plan, and once the isoparametric elements had been demonstrated it was agreed that they should be retained. The problem of Cauchy principal values was solved by not calculating them. The simultaneous equations were equations of nodal collocation as in BITEMJ, and nodes of quadratic isoparametric elements could be at corners of the boundary. An equation of collocation is of the form

$$
c_{i j}(x) u_{j}(x)+\int_{S} T_{i j}(x, y) u_{j}(y) \mathrm{d} S_{y}=\int_{S} U_{i j}(x, y) t_{j}(y) \mathrm{d} S_{y}
$$

where the Cauchy principal value of the integral on the left side is taken, and $c_{i j}(x)$ is the limiting value of an integral over that part of the surface of a region of exclusion $v(x, \varepsilon)$ which lies inside the domain of analysis $V$ bounded by $S$. For $x$ at a corner, $c_{i j}(x) \neq \delta_{i j} / 2$. It is possible to compute separately $c_{i j}(x)$ and the Cauchy principal value, but one may ask whether it is necessary given that whereas both quantities depend upon the arbitrarily chosen shape of $v(x, \varepsilon)$, the physical solution of the boundary value problem is independent of that shape. The simpler alternative became apparent upon consideration of the computational procedure. A leading diagonal submatrix of equation coefficients is the sum of $c_{i j}(x)$, and integrals of $T_{i j}(x, y)$ multiplied by shape functions which equal 1.0 at $x$. All other kernel-shape function products are at most weakly singular at $x$ and therefore may be integrated by Gaussian quadrature formulae. Finally, surface tractions $t_{j}(y)$ due to rigid body translations of the domain $V$ equal zero. The procedure is therefore to evaluate by Gaussian quadrature all equation coefficients except for those of the leading diagonal submatrix (the explicitly computed component), then by consideration of rigid body translations compute the leading diagonal submatrix (the implicitly computed component). An application of the program for plane strain to the computation of stress intensity factors for an ASTM compact tensile specimen is described by Boissenot, Lachat and Watson [11].

The program for three dimensional analysis (EITD) was intended to be as versatile as the finite element program that had been written earlier. In particular it was to be capable of analysing piecewise homogeneous domains, so the need arose for interface elements, at nodes of which both displacements and interface tractions are unknown. To achieve economical and robust evaluation of integrals, numbers of Gauss points were chosen according to upper bounds for error due to Stroud and Secrest [6]. In practice this meant that, for each available Gaussian formula, there was an upper limit on the ratio of length of interval over which it was applied to minimum distance from the interval to the collocation point. Program EITD ran on a CDC7600, capable of about 5 megaflops but with just 64K of word addressable memory (SCM), 60 bits per word. The upper limit on subregion (homogeneous subdomain) mesh size was 75 quadratic isoparametric elements: this could probably have been increased to 150 without unacceptable disc file input-output time. In the solution of larger problems, it was necessary artificially to subdivide the domain of analysis into subregions to stay within problem size limits $[12,13]$. This is undesirable, because displacements at points inside the domain of analysis which lie on interfaces are constrained to vary only as permitted by the interpolations over interface elements.

Zienkiewicz occasionally visited CETIM to give lectures on finite elements. He took an interest in our development of boundary elements, and later initiated research at 
Swansea into coupling of the methods. Coupling is intended to make best use of both methods, especially in nonlinear analysis for which it has generally been considered that boundary elements are unsuitable. There was occasional contact with Cruse at Pratt and Whitney Aircraft, and at some time after my return to UK in 1975 a copy of EITD found its way across the Atlantic. This was subsequently modified by Wilson and Cruse [14] to analyse problems for anisotropic elastic material. After both Lachat and myself had left CETIM, the development of boundary elements came to an end.

\section{Imperial College}

My intention in returning to UK was to enter the mainstream civil engineering industry. However, once the results of the work at CETIM had been published I was contacted by two departments of Imperial College, Mathematics and Mineral Resources Engineering. The Department of Mineral Resources Engineering obtained for me a research fellowship, and I joined IC in 1978. For mining engineers, an attraction of boundary elements was the ease with which they could be used to analyse problems of infinite or semi-infinite extent. Surfaces and interfaces between different materials could also be of infinite extent, so in Program THREE were implemented, in addition to the quadrilateral and triangular elements of EITD, infinite boundary elements with displacement either tending to zero at infinity, or corresponding to states of plane and antiplane strain. The latter type of infinite element is useful in the analysis of problems which are only locally three dimensional, such as tunnel intersections.

At this stage it seemed appropriate to ask the question, was this new method of stress analysis successful? All depends upon what is considered to be success, and different people will give different answers. Since engineering is not a fine art, success should be measured according to industrial rather than academic criteria and the answer should perhaps be no. Almost all numerical modelling in solid mechanics was being carried out by the finite element method, as implemented in packages such as NASTRAN, ADINA and MARC. The next question to ask was, why? One reason was that a vast amount of effort had been put into overcoming the inconveniences of finite element analysis, principally by means of elaborate pre- and postprocessing graphics. Since there is no point in re-inventing the wheel, an answer might be to team up with a provider of such graphics, and interface their software to boundary element analysis. That is only possible if the boundary element method has credibility, so firstly it has to be demonstrated that boundary elements will solve problems of practical size (as opposed to academic examples) more economically than finite elements. Since it was not clear that Program THREE could do that, attention turned to the achievement of further improvements of performance.

Experience indicated that linear elements are more efficient than constant, and quadratic are more efficient than linear. For large problems, simultaneous equation solution takes far more computer time than any other part of a boundary element analysis, so every effort must be made to minimise the total number of degrees of freedom. Increases in the degree of functional variation over each element result in a preponderance of nodes on sides of serendipity quadrilaterals, and of nodes both on 
sides of and inside Lagrangian quadrilaterals. Whereas nodes and so degrees of freedom at corners are shared by about four neighbouring elements, nodes on sides are shared by only two and interior nodes are not shared at all. The total number of degrees of freedom therefore increases rapidly. It is preferable to concentrate all the degrees of freedom at corners of elements, and one way to achieve this is to construct shape functions from Hermitian polynomials [15]. The degrees of freedom of Hermitian cubic elements include tangential derivatives of displacement, and on a smooth part of the surface computed displacements are $\mathrm{C}^{1}$ continuous between elements. The immediate reason for a move to Hermitian elements, though, was to reduce the total number of degrees of freedom, so that for example if a mesh of quadratic elements were replaced by the same number of Hermitian cubic elements, the total number of degrees of freedom would be unchanged despite the substitution of cubic for quadratic functional variation over each element. In practice, satisfactory accuracy could then be achieved using a coarser mesh.

As what was intended to be a preliminary exercise, a program for the analysis of plane strain was developed. There would be four unknowns at each node, but the usual method of nodal collocation would yield only two equations. Two schemes were considered: a Galerkin formulation in which each of the simultaneous equations would be a linear combination of equations of collocation at Gauss points of elements, and nodal collocation of both equation (1) and the hypersingular equation obtained by tangential differentiation of equation (1). The latter scheme was chosen and the two integral equations were written in the form

$$
\begin{aligned}
& \lim _{\varepsilon \rightarrow 0} \int_{S-S(x, \varepsilon)+s(x, \varepsilon)}\left[T_{i j}(x, y) u_{j}(y)-U_{i j}(x, y) t_{j}(y)\right] \mathrm{d} S_{y}=0 \\
& \lim _{\varepsilon \rightarrow 0} \int_{S-S(x, \varepsilon)+s(x, \varepsilon)}\left[V_{i j}(x, y) u_{j}(y)-W_{i j}(x, y) t_{j}(y)\right] \mathrm{d} S_{y}=0
\end{aligned}
$$

where $S(x, \varepsilon)$ is the part of the surface of the region of exclusion $v(x, \varepsilon)$ which lies on $S$ (and would be excluded in the definition of a Cauchy principal value), and $s(x, \varepsilon)$ is the part of the surface of $v(x, \varepsilon)$ which lies inside $V$. The kernels $V_{i j}(x, y)$ and $W_{i j}(x, y)$ are the tangential derivatives of $T_{i j}(x, y)$ and $U_{i j}(x, y)$, and it is understood that the differentiations take place before $\varepsilon$ tends to zero. In equation (3), $V_{i j}(x, y)$ is hypersingular and $W_{i j}(x, y)$ is strongly singular like $T_{i j}(x, y)$. Equation (2) is equivalent to equation (1), and for equation (3) the implicitly computed component is taken to include the integral over $s(x, \varepsilon)$, integrals of $V_{i j}(x, y)$ multiplied by shape functions the value or tangential derivative of which equals 1.0 at $x$, and integrals of $W_{i j}(x, y)$ multiplied by shape functions which equal 1.0 at $x$. By consideration of a Taylor series expansion, it can be shown that the implicitly computed component of equation (3) is of the form

$$
a_{i j}(x) u_{j}(x)+b_{i j}(x) w_{j}(x)+c_{i j}(x) t_{j}(x)
$$


where $u_{j}(x), w_{j}(x)$ and $t_{j}(x)$ are displacement, tangential derivative of displacement and traction at $x$ respectively. Just as for equation (2) the coefficients of the leading diagonal submatrix are determined by consideration of rigid body translations, so for equation (3) the coefficients $a_{i j}(x), b_{i j}(x)$ and $c_{i j}(x)$ are computed by consideration of trial displacement fields, these being rigid body translations and rotations, and displacements due to uniform stress fields. This procedure is exactly that later referred to as the method of simple solutions [16].

For the program to be useful, it would be necessary to develop special elements for the modelling of re-entrant corners, which are common in mines. Already with quadratic elements the computed results near such corners were poor, and with Hermitian cubic elements they would be worse. According to the theory of elasticity, stresses tend to infinity at notch roots [17]. In Program PLANE, therefore, additional shape functions which exhibit the same singular behaviour as the theoretical solution were defined over elements adjacent to notch roots. These functions multiply stress intensity factors, the values of which are computed simultaneously with nodal displacements and tractions. From this development followed one of interest to the wider engineering community: a crack is the limiting case of a notch, so PLANE could also compute directly, without any form of postprocessing, stress intensity factors at crack roots [18].

\section{Mount Isa Mines}

In 1982, the slow progress on Hermitian elements was interrupted by an opportunity not to be missed: an Australian mining company wished to obtain a licence to run THREE. There was a problem. MIM were quite impressed by THREE, but they wanted to carry out analyses somewhat larger than anything previously envisaged. Stresses were to be computed in rock surrounding at least 30 stopes (underground cavities) of various shapes. There was no time to develop Hermitian cubic elements for three dimensional analysis. Someone, possibly Gernot Beer who was advising MIM in the matter, put forward an idea that I had once pondered but never acted upon, to lump offdiagonal submatrices of small equation coefficients to yield a sparsely populated matrix.

In the absence of estimates of error due to lumping, it was deemed prudent to use the approximate lower and upper triangular factors obtained by bifactorisation of the lumped matrix to compute search vectors in an iterative solution [19]. If the iteration converged, the results would be the same as those which would have been obtained without lumping. Fortunately this worked and there only remained to develop a version of THREE that would run on MIM's Unisys machine, with as large problem size limits as possible. This was not easy, as THREE incorporated much CDC-specific code which for optimal performance had to be replaced by equivalent Unisys-specific code. The program also had to fit into $256 \mathrm{~K}$ of 36 bit words. In the initial version the problem size limit was 250 elements per subregion, and in a later version 400 elements. This was not enough to model 30 stopes, but adequate for most purposes. 


\section{Boundary elements from 1980 to the present day}

Research into boundary elements perhaps reached its height between 1980 and 1990. The systems BEASY (UK), BETSY (Germany) and BEST3D (USA) were under development during this period. Several textbooks were written, and since an apparent disadvantage of boundary elements was that they could only be used to solve efficiently linear problems, much effort was expended on transient and nonlinear analysis. Mathematicians such as Wendland [20] sought to substantiate the work of engineers by rigorous analysis, but not many engineers were sufficiently well versed in mathematics to understand the significance of their conclusions. The indirect formulation was largely forgotten, except under the guises of panel methods in aerodynamics [21], the displacement discontinuity method for slots left by mining of tabular orebodies, and the body force method used to calculate many of the results presented in compendia of stress intensity factors such as that compiled by Murakami [22].

The Galerkin method came into favour, and certainly for a given mesh it is more accurate than nodal collocation. In some respects the numerical implementation is simpler, but in an efficient computational scheme there must be a scatter process whereby factored equations of collocation at Gauss points are added to equations indexed on nodes. This introduces complications if, as is likely to be the case for large problems, the equations cannot be held in byte addressable memory. At one stage there was interest in Overhauser elements, the shape functions of which are constructed from B-splines. These elements offer $\mathrm{C}^{2}$ interelement continuity of displacement, and rate very well by the measure of degrees of freedom per element, but there are onerous restrictions on the topology of a mesh in three dimensional analysis. By contrast, there are no restrictions on the way in which Hermitian cubic elements are assembled to form a mesh, because each element is stand-alone like a piece of Lego.

Hypersingular integral equations assumed an important role in the analysis of cracks, for which the singular equation alone does not provide enough information to calculate displacements of both faces. The hypersingular equation usually chosen is the traction equation, but there are other possibilities. The evaluation of hypersingular integrals is something of a challenge: methods that have been proposed include Hadamard finite parts, simple solutions as referred to earlier, and the application of Stokes' theorem. Simple solutions are to be preferred, because boundary element methods are complicated enough already.

The version of Program PLANE described earlier could only analyse cracks in planes of symmetry. The current version analyses cracks of arbitrary geometry, by reference to equations (2), (3) and two more hypersingular equations obtained by differentiation of equations (2) and (3) in the direction normal to a crack. Subdominant modes of crack opening displacement are taken into account, and the singular shape functions which multiply stress intensity factors extend over many elements on each face of the crack [23]. Program THREE now does everything that is done by PLANE, except in that the elements are still quadratic [24]. Although Hermitian cubic elements are not the complete answer to the problem of simultaneous equation solution time, they have performed very well in other respects and their incorporation into THREE is high on 
the wish list. The iterative solution procedure of THREE does not perform so reliably in the analysis of finite body problems as in that of infinite domains. Attempts are now being made to develop a robust iterative scheme, as therein probably lies the key to economical solution of very large problems.

From about 1990 onwards, progressively larger analyses could be carried out on a personal computer instead of a mainframe. A PC is just a mainframe on a desk, but software that ran efficiently on a mainframe did not necessarily do so on a PC. Mainframe word or byte addressable memory was expensive and therefore of very limited capacity, but to compensate for that, the data transfer rate between that memory and disc storage was high and it was usually possible to perform simultaneously arithmetic and input-output by means of double buffering. On a PC, RAM is cheap so there is more of it, whereas input-output to disc is relatively slow. It required considerable effort to adapt matrix construction and simultaneous equation solution schemes to perform well in the new environment. In Program THREE, the work that had been done to optimise vectorisation on the Cray turned out to be largely a waste of time. Fortran 90, in which principles of object oriented programming are formalised, was introduced. OOP is a laudable aim but, to the annoyance of those of us who were at ease programming in a language with practically no safeguards and few strictly enforced rules, some useful tricks were not allowed in the new scheme of things. Nevertheless, the Fortran 90 manual does describe a logical framework which can be used to advantage when writing Fortran 77. There are many home-made objects in THREE and PLANE.

\section{The future}

Success in the sense of widespread industry acceptance still eludes the boundary element community, but the day when the computational advantages over finite elements in some applications become too attractive for end users to ignore may not be far away. The early view that boundary elements must be superior because they reduce the dimension of the numerical problem to be solved was rather simplistic. Matrices of equation coefficients generated by boundary element methods are fully populated, and also some finite element solvers now in use are several times faster than the original frontal solver of Irons.

But boundary elements have other advantages. It is easier to generate mesh over a surface than through a volume. In a boundary element analysis, the governing differential equation is exactly satisfied at every point of the domain, so the mesh of boundary elements required to achieve satisfactory accuracy is coarser than that of finite elements with the same degree of functional variation. Furthermore, the maximum stresses are usually at the surface of a body subjected to load, and at stress concentrations, the rate at which stresses increase as the surface is approached is invariably high. In the finite element method, interpolation within elements imposes constraints upon the computed variation of displacement and stress as the surface is approached, so unless the mesh is very fine, the computed surface stresses are too low. This effect is compounded by the need to calculate stresses at Gauss points and then 
extrapolate to the surface. In boundary element analysis, these sources of error do not exist.

It was suggested earlier that input data for boundary element software might be generated by an existing interactive graphical package. In recent years, efforts have been made to integrate seamlessly the entire CAD-CAM process of initial development of a concept, analysis and refinement of the design, and numerically controlled manufacture. It is now commonly expected, for example, that software for engineering analysis will take as input data a faceted model of an object, which has been generated from the original data created by a drafting package. The faceted model, often defined by an IGES file, typically consists of flat triangular patches and is not an exact representation of the proposed design. In finite element analysis, a mesh of four node tetrahedral elements is usually then generated through the volume of the object. Theoretically there are stress singularities at artificial notch roots between facets, but the computed stresses are uniform within each tetrahedral element and so the presence of singularities is not reflected in the results. Even if quadratic finite elements are used, computed results are still reasonably smooth. Boundary element methods, however, try to compute faithfully the local variations of stress resulting from the imperfections of the faceted model, and the user then complains about curious peaks of stress on smooth surfaces. Of course the fault lies not with the boundary element method, but with the faceted model. The ideal remedy would be to have the boundary element software take as input the exact geometric model from the drafting package, and generate the mesh directly from that data. A less satisfactory solution is to read the IGES file, and try to determine which edges and notch roots of the faceted model are artificial and which are real, in an attempt to recreate the original geometry.

Most of us who develop boundary elements have a feel for how a mesh should be graded to yield the best possible results for a given number of elements. It may be a surprise to many of us, but this intuitive ability is not a common human attribute, even among engineers in industry. There is therefore a need for automatic mesh gradation, according to criteria that might even be derived a priori from the boundary integral equation itself. Development of such criteria may require some understanding of functional analysis, the results of which are generally expressed in a mathematical language few engineers understand.

Researchers into boundary elements have tended to advance on a wide front, as if the primary objective were to demonstrate that boundary elements, like finite elements, can do practically everything. If we wish to encourage the widespread use of boundary elements in industry, we should identify and concentrate our efforts on applications in which they have a special advantage. Fracture mechanics is an obvious candidate. Very little explicit modelling of cracks is currently carried out by the finite element method, because it is difficult. Instead, estimates of stress intensity factors and fatigue life are made by postprocessing software, on the basis of computed stresses in uncracked components. It is, though, quite practical to model cracks explicitly by boundary element methods. Moreover, it should be easy to introduce into boundary element analysis cohesive elements and plastic zones at crack roots. Boundary 
elements also have an advantage in moving boundary problems and optimisation, because it is easier to modify a surface mesh than to modify one through the volume.

\section{Conclusion}

The development of boundary element methods has been under way for forty years, but the number of researchers in the field has always been relatively small. Early progress could have been more rapid, had there been a greater awareness of numerical techniques being used by others. The direct formulation, as first proposed by Jaswon, Ponter and Symm and then applied to elasticity by Rizzo and Cruse, is not necessarily more accurate or efficient than the indirect formulation, but it is easier to implement and for that reason it is the more widely used.

Whereas in the early stages it was considered by some that boundary elements would be easier to implement than finite elements, it is now known that the reverse is true. The rate of progress continues to be limited not by any lack of mathematical ingenuity, but by a scarcity of researchers capable of turning the mathematics into efficient software. As a result, the common perception has become that boundary elements are a good idea in principle but not in practice. That can be changed, especially if at first we concentrate our efforts on niche applications for which the comparison with finite elements is particularly favourable.

\section{References}

1. Jaswon, MA and Ponter, AR: An integral equation solution of the torsion problem, Proc. Roy. Soc. Series A 273, 237 (1963).

2. Jaswon, MA: Integral equation methods in potential theory 1, Proc. Roy. Soc. Series A 275, 23 (1963).

3. Symm, GT: Integral equation methods in potential theory 2, Proc. Roy. Soc. Series A 275, 33 (1963).

4. Muskhelishvili, NI: Some Basic Problems of the Mathematical Theory of Elasticity, Noordhoff, Groningen (1953)

5. Kupradze, VD: Metody Potensiala v Teorii Uprugosti, Fizmatgiz, Moscow (1963).

6. Stroud, AH and Secrest, D: Gaussian Quadrature Formulas, Prentice-Hall, Englewood Cliffs (1966).

7. Watson, JO: The Analysis of Thick Shells with Holes, by Integral Representation of Displacement, $\mathrm{PhD}$ thesis, University of Southampton (1972).

8. Rizzo, FJ: An integral equation approach to boundary value problems of classical elastostatics, Q. Appl. Math. 25, 83 (1967).

9. Cruse, TA: Numerical solutions in three dimensional elastostatics, Int. J. Solids Structures 5, 1259 (1969).

10. Riccardella, PC: An Improved Implementation of the Boundary Integral Technique for Two Dimensional Elasticity Problems, Carnegie-Mellon Intitute of Technology, Pittsburgh (1972). 
11. Boissenot, JM, Lachat, JC and Watson, JO: Etude par équations intégrales d'une éprouvette CT15, Revue de Physique Appliquée 9, 611 (1974).

12. Lachat, JC: A Further Development of the Boundary Integral Technique for Elastostatics, $\mathrm{PhD}$ thesis, University of Southampton (1975).

13. Lachat, JC and Watson, JO: Effective numerical treatment of boundary integral equations: a formulation for three dimensional elastostatics, I. J. Num. Meth. Engg. 10, 991 (1976).

14. Wilson, RB and Cruse, TA: Efficient implementation of anisotropic three dimensional boundary integral equation stress analysis, I. J. Num. Meth. Engg. 12, 1383 (1978).

15. El-Zafrany, A and Cookson, RA: Derivation of Lagrangian and Hermitian shape functions for quadrilateral elements, I. J. Num. Meth. Engg. 23, 1939 (1986).

16. Lutz, E, Ingraffea, AR and Gray, LJ: Use of 'simple solutions' for boundary integral equation methods in elasticity and fracture analysis, I. J. Num. Meth. Engg. 35, 1737 (1992).

17. Williams, ML: Stress singularities resulting from various boundary conditions in angular corners of plates in extension, J. Appl. Mech. ASME 74, 526 (1952).

18. Watson, JO: Hermitian cubic elements for plane problems of fracture mechanics, Res Mechanica 4, 23 (1982).

19. Beer, G and Watson, JO: Introduction to Finite and Boundary Elements for Engineers, Wiley, Chichester (1992).

20. Wendland, WL, Stephan, E and Hsaio, GC: On the integral equation method for the plane mixed boundary value problem of the Laplacian, Math. Meth. Appl. Sci. 1, 265 (1979).

21. Hunt, B and Hewitt, BL: The indirect boundary integral formulation for elliptic, hyperbolic and nonlinear flows. In Banerjee, PK and Watson, JO (editors): Developments in Boundary Element Methods - 4, Elsevier Applied Science Publishers (1986).

22. Murakami, Y: Stress Intensity Factors Handbook, Pergamon, Oxford (1987).

23. Watson, JO: Singular boundary elements for the analysis of cracks in plane strain, I. J. Num. Meth. Engg. 38, 2389 (1995).

24. Watson, JO: Singular boundary elements for cracks and notches in three dimensions. In Mang, RA, Rammerstorfer, FG and Eberhardsteiner, J (editors): Proc. Fifth World Congress on Computational Mechanics, ISBN 3-9501554-0-6, http://wccm.tuwien.ac.at, Technical University of Vienna (2002). 